\title{
SITE CHARACTERISATION: ASTRONOMICAL SEEING FROM A TURBULENCE-RESOLVING MODEL
}

\author{
M. NICKOLA \\ Space Geodesy Programme, Hartebeesthoek Radio Astronomy Observatory, \\ P.O. Box 443, Krugersdorp 1740, South Africa \\ e-mail: marisa@hartrao.ac.za \\ R.C. BOTHA \\ Space Geodesy Programme, Hartebeesthoek Radio Astronomy Observatory, \\ P.O. Box 443, Krugersdorp 1740, South Africa \\ e-mail: roelf@hartrao.ac.za
}

\section{ESAU}

G.C. Rieber Climate Institute at the Nansen Environmental and Remote Sensing Center, Thormohlensgt. 47, 5006, Bergen, Norway

e-mail: igore@nersc.no

\section{G.D. DJOLOV}

University of Pretoria, Department of Geography, Geoinformatics and Meteorology, Faculty of Natural and Agricultural Sciences, University of Pretoria 0002, South Africa e-mail: george.djolov@up.ac.za

\section{W.L. COMBRINCK}

Space Geodesy Programme, Hartebeesthoek Radio Astronomy Observatory, P.O. Box 443, Krugersdorp 1740, South Africa e-mail: ludwig@hartrao.ac.za@

\section{December Geological Society of South Africa}

\begin{abstract}
A Lunar Laser Ranging (LLR) system is to form part of geodetic instrumentation to be located at a new fundamental space geodetic observatory for South Africa. For optimal efficiency, LLR requires optical resolution or so-called astronomical seeing conditions of $\sim 1$ arc-second in order to deliver usable ranging data. Site characterisation should include a description of astronomical seeing for various locations on-site and overall atmospheric conditions. Atmospheric turbulence degrades astronomical seeing. In-situ methods of determining astronomical seeing are difficult, time-consuming and costly. We propose the use of a turbulence-resolving model to determine and predict astronomical seeing at a site. Large Eddy Simulation NERSC (Nansen Environmental and Remote Sensing Centre) Improved Code (LESNIC) is a turbulence-resolving simulation code which models atmospheric turbulence. It has been used to compile a database of turbulence-resolving simulations, referred to as DATABASE64. This database consists of a collection of LESNIC runs for a stably stratified planetary boundary layer (SBL) over a homogeneous aerodynamically rough surface. Results from DATABASE64 for the nocturnal boundary layer are employed to render profiles of the vertical distribution of optical turbulence ( $C_{N}^{2}$ profiles). Seeing parameter values are also obtained by making use of DATABASE64 results. The $C_{N}^{2}$ profiles and seeing parameter values obtained from DATABASE64 results are compared with general observational results that have been published in the literature. The values obtained are consistent with results from field campaigns as reported. Turbulence-resolving models, such as LESNIC, show potential for delivering and predicting profiles and parameters to characterise astronomical seeing, which are essential prerequisites for establishing an LLR system at the most suitable site and most suitable on-site location. A two-pronged approach is envisaged - in addition to modelling, quantitative seeing measurements obtained with an on-site seeing monitor will be used to verify and calibrate results produced by the LESNIC model.
\end{abstract}

\section{Introduction}

A new fundamental space geodetic observatory to deliver high quality and quantity geodetic data is planned for South Africa. A Lunar Laser Ranging (LLR) system is under development and will be located at a still to be determined site. The LLR's laser beam becomes diverged and the beam energy profile is adversely affected during propagation from the Earth to the Moon and back. This is as a result of turbulence in the atmosphere that causes dispersion and divergence of the laser beam. The LLR technique requires optimal optical seeing conditions at $\sim 1$ arc-second resolution 
level to achieve millimetre-level accuracy in ranging to the Moon. Determining optical seeing conditions for various locations requires on-site measurements which form an integral part of site characterisation (Combrinck et al., 2007). In-situ methods of determining optical (commonly known as astronomical) seeing conditions are difficult, time-consuming and costly. A turbulenceresolving model to determine and predict astronomical seeing at a site is proposed.

\section{Atmospheric turbulence}

Atmospheric turbulence in the layer closest to Earth, the planetary boundary layer (PBL), contributes significantly to the degradation of optical seeing quality. The PBL is directly influenced by interaction with the ground and, depending on time of day and weather conditions, varies from ground level to an altitude of $\sim 200 \mathrm{~m}$ to $2 \mathrm{~km}$ (Trinquet et al., 2006). Atmospheric turbulence results when the boundary layer between air masses with different temperatures breaks up into local unstable air masses known as eddies. Eddies act as weak, irregular lenses with varying refractive indices, distorting wave fronts. Eddies have scale sizes ranging from large to small. The large scale size is referred to as the outer scale of turbulence, $\mathrm{L}_{0}$, and ranges from tens to hundreds of meters, with wind shear or convection as energy source. The small scale size is referred to as the inner scale of turbulence, $l_{0}$, and is on the order of a few millimetres in size (Stull, 1988).

In the Kolmogorov model of turbulence, energy is added to the largest scale eddies and transferred to progressively smaller scale eddies to be dissipated by viscous action at the smallest scales (Pope, 2000). This cascade process is responsible for variations in temperature and density, which leads to refractive index variations. A laser beam will thus be significantly degraded as it travels through these turbulent layers with fluctuating refractive indices. The effects of turbulence on the propagation of a laser beam from the earth to the moon and back again may be found by determining the vertical distribution of turbulence (Trinquet et al., 2006). This vertical distribution of turbulence, also known as the profile of the refractive index structure constant, $C_{N}^{2}$, may be obtained by making use of statistical parameters from the Kolmogorov model of turbulence (Kolomogorov 1941), as developed by Tatarski (Tatarski 1961), and the Gladstone relation (Bean and Dutton, 1966):

$$
C_{N}{ }^{2}(h)=\left(80 \times 10^{-6} \frac{P(h)}{T^{2}(h)}\right)^{2} C_{T}^{2}(h)
$$

The profile of turbulence strength as a function of altitude, $C_{N}^{2}(b)$, where $b$ is the height above the telescope, may thus be obtained by measurement of the atmosphere's temperature profile, $C_{T}^{2}(b)$, as well as meteorological parameters: pressure $P[\mathrm{hPa}]$ and absolute temperature, $T[\mathrm{~K}]$.
The integrated value of the refractive index structure constant, $C_{N}^{2}$, allows for predicting atmospheric optical quality in terms of astronomical seeing (Abahamid 2004).

\section{Astronomical seeing}

The theoretical resolution limit of a telescope is given by the Rayleigh criterion:

$$
\theta=1.22 \frac{\lambda}{D}
$$

where $\theta$ is the angular resolution, $\lambda$ the wavelength of incoming light and $D$ the telescope aperture (Argyle, 2004). A telescope's effective theoretical angular resolution is furthermore also limited by atmospheric seeing conditions. Seeing can therefore be determined by measuring the smallest resolvable angular resolution of an object outside of Earth's atmosphere.

Astronomers quantify the quality of optical seeing conditions at a particular site with a parameter they refer to as the "seeing". Seeing, $\varepsilon_{F W H M}$, is described in terms of the full width at half maximum (FWHM) of a star's intensity distribution at the focus of a telescope. Seeing is related to a statistical parameter provided by the Kolmogorov model of turbulence (Kolmogorov, 1941), as developed by Fried (Fried, 1965 and 1966), called the Fried parameter, $r_{0}$ :

$$
\varepsilon_{F W H M}=0.98 \frac{\lambda}{r_{0}}
$$

where $\lambda$ is the wavelength of observation. The Fried parameter also provides a meastre of image degradation due to atmospheric turbulence. A larger $r_{0}$ results in a smaller $\varepsilon_{F W H M}$, indicating better seeing. At the best sites, $r_{0}$ is $\sim 20 \mathrm{~cm}$. The Fried parameter indicates the diffraction-limited aperture, $r_{0}$, that will produce the same angular resolution as in the seeing-limited case (Quirrenbach, 2006).

The profile of turbulence strength as a function of altitude, also known as the index of refraction structure parameter, $C_{N}^{2}(b)$, is related to the seeing through this Fried parameter:

$$
r_{0}=\left(0.423\left(\frac{2 \pi}{\lambda}\right)^{2} \int_{0}^{\infty} C_{N}^{2}(h) d h\right)^{\frac{-3}{5}}
$$

where the turbulence strength $C_{N}^{2}(b)$ varies as a function of height $b$ above the telescope (Roddier, 1981). The expression for seeing thus becomes (Vernin and Muñoz-Tuñón, 1992):

$$
\varepsilon_{F W H M}=0.98 \frac{\lambda}{r_{0}}=5.25 \lambda^{\frac{-1}{5}}\left(\int_{0}^{\infty} C_{N}^{2}(h) d h\right)^{\frac{-3}{5}}
$$


From Equation (5), the inclusion of a turbulenceresolving model to obtain $C_{N}^{2}$ profiles for a specific site, allows one to estimate seeing at the site.

\section{Turbulence-resolving model}

Atmospheric turbulence may be simulated with numerical modelling, such as with the Large Eddy Simulation NERSC (Nansen Environmental and Remote Sensing Centre) Improved Code (LESNIC), which is a turbulence-resolving simulation code (Esau, 2004). LESNIC is based on the Kolmogorov model of turbulence, a statistical analysis of turbulence (Kolmogorov, 1941 and Tatarski, 1961). With LESNIC, the PBL's dynamics, thermodynamics and interaction with the underlying surface are described by a set of differential equations and appropriate boundary conditions from fluid mechanics. Local unstable air masses (eddies) are either resolved or modelled. Largescale eddies are resolved through solving partial differential equations governing turbulent fluid flow, the Navier-Stokes equations. Small-scale eddies dissipate the energy through viscous interactions. They cannot be resolved explicitly and are filtered out numerically. Being more isotropic than the larger scale eddies, these small-scale eddies' influence on the flow are parameterised and may be modelled with a universal subgrid-scale model (Stull, 1988 and Garratt, 1995).

The large-scale eddy simulation code LESNIC developed at G.C. Rieber (Climate Institute at the Nansen Environmental and Remote Sensing Center (Esau, 2004)) has been used to compile a database of turbulence-resolving simulations, referred to as DATABASE64. The code numerically solves NavierStokes equations of motions for an incompressible Boussinesq fluid with small density differences, as well as the transport equations for the potential temperature and passive scalars. This database consists of a collection of LESNIC runs for an SBL over a homogeneous aerodynamically rough surface. The runs simulate a period of 16 hours of PBL turbulence. These runs produce three-dimensional fields of fluctuations of the potential temperature as well as three Cartesian components of the wind velocity, $u, v$ and $w$. Turbulence statistics and horizontally time-averaged profiles of required quantities are obtained by further processing of the above mentioned fluctuations (Esau, 2004). Results from DATABASE64 for the nocturnal boundary layer are employed to render profiles of the vertical distribution of optical turbulence ( $C_{N}^{2}$ profiles) and to obtain seeing parameter values.

\section{Results and discussion}

LESNIC runs from DATABASE64 for a stably stratified planetary boundary layer (SBL) over a homogeneous aerodynamically rough surface were used to obtain vertical profiles of $C_{N}^{2}$ as well as the Fried parameter, $r_{0}$, and seeing, $\varepsilon_{F W H M}$.

Profiles of $C_{N}^{2}$ were obtained by making use of the Gladstone relation, Equation (1). The $C_{T}^{2}$ parameter was determined from rates of temperature variance dissipation, $\varepsilon_{\theta}$, and turbulence kinetic energy dissipation, $\varepsilon^{-1 / 3}$, in accordance with (André et al., 1978):

$$
C_{T}^{2}=1.6 \varepsilon_{\theta} \varepsilon^{-1 / 3}
$$

where $\varepsilon_{0}, \varepsilon^{-1 / 3}, P$ and $T$ were provided by DATABASE64 for the specific run.

The Fried parameter, $r_{0}$, was obtained from Equation (4) by integrating $C_{N}^{2}$ with respect to zenith. The seeing, $\varepsilon_{F W H M}$, follows from Equation (5).

The simulation produced the profile in Figure 1 and a seeing parameter value of $r_{0}=5 \mathrm{~cm}$ and $\varepsilon_{\text {FWHM }}=2.1$ ".

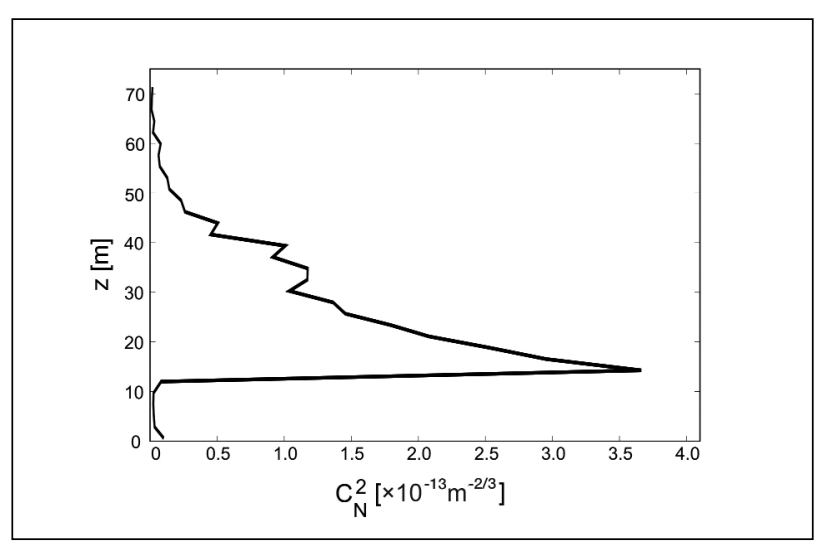

Figure 1. Simulated $C_{N}^{2}$ profile obtained from one of the LESNIC runs from DATABASE64.

The $C_{N}^{2}$ profile and seeing parameter values obtained from DATABASE64 were then compared with observational results from the literature.

Figure 2 shows shows the profile observed for the first $80 \mathrm{~m}$ above ground at Dome $\mathrm{C}$ in Antarctica during the winter of 2005 . Dome $\mathrm{C}$ is located at latitude $74.5^{\circ} \mathrm{S}$ on top of a local maximum of the Antarctic plateau, 3250 $\mathrm{m}$ above mean ice level. Data was obtained by balloon radio sounding observations (Aristidi et al., 2009).

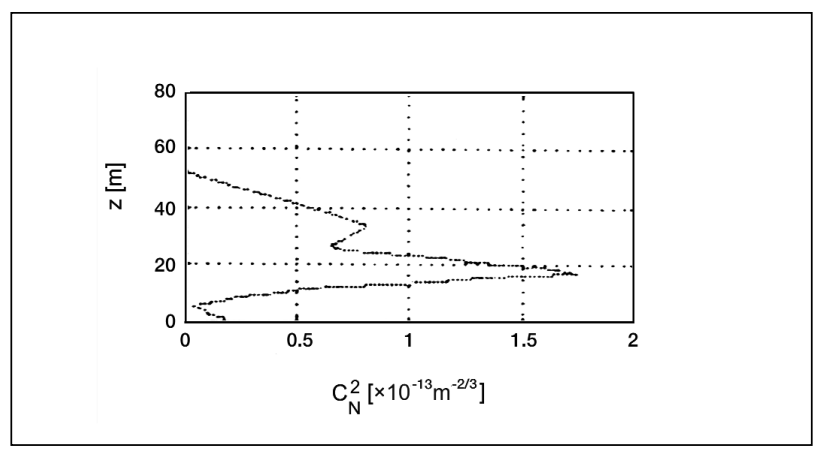

Figure 2. Observed $C_{N}^{2}$ profile obtained from observations by balloon radio sounding (Aristidi et al., 2009). 
This profile was one of 32 profiles obtained during an astronomical site testing campaign at Dome $\mathrm{C}$ in the austral winter (polar night - June 21 to September 21, 2005). The Antarctic plateau is essentially free of topographic features. Flights of meteorological balloons, equipped with microthermal sensors to measure thermal fluctuations $(\mathrm{mK})$, were used to determine the vertical profile of the optical turbulence intensity, $C_{N}^{2}$, from the ground up to $20 \mathrm{~km}$.

The balloon transmits the value of the refractive index structure constant every 1 to 2 seconds, which corresponds to a vertical resolution of 5 to $10 \mathrm{~m}$. Meteorological parameters such as pressure, temperature, humidity and wind speed components were also provided by the soundings. The specific profile was obtained during flight number 563 , launched on the 2nd of September 2005

(Aristidi et al., 2009 and Trinquet et al., 2008). Seeing results for two elevations $(8 \mathrm{~m}$ and $33 \mathrm{~m})$ retrieved from balloon $C_{N}^{2}$ profiles (Trinquet et al., 2008):

$\varepsilon_{\text {FWHM }}=1.9 "$ and $r_{0} \approx 5 \mathrm{~cm}(8 \mathrm{~m})$

$\varepsilon_{F W H M}=0.9 "$ and $r_{0} \approx 11 \mathrm{~cm}(33 \mathrm{~m})$

The quantitative shapes of the simulated and observed profiles correlate well. Seeing parameter values obtained from the model are in close agreement with observed values.

\section{Conclusions and Recommendations}

Seeing is one of the important characteristics in determining whether a site is suitable for a space geodetic station, as the seeing conditions determine the quality and quantity of data. In-situ methods of determining astronomical seeing are difficult, timeconsuming and costly in terms of equipment. The LESNIC model seems capable of reproducing observed $C_{N}^{2}$ profiles and delivering seeing parameter values consistent with values measured during field campaigns. By making use of site-specific data for initial and boundary conditions as well as for topographic features and surface roughness, a turbulence-resolving model such as LESNIC may potentially be used to deliver and predict $C_{N}^{2}$ profiles, which can then be used to determine astronomical seeing conditions as part of site characterisation.

Optical seeing conditions and atmospheric turbulence can be connected by combining methods from astronomical seeing and boundary layer meteorology. Optical seeing conditions may be determined by implementation of an automated seeing monitor on-site. Modelled results (with meteorological conditions as input) can then be compared with quantitative seeing measurements to determine whether a good correlation exists between the LESNIC model's predicted results and actual seeing quality at a site. It will allow for determining the relationship between seeing quality and meteorological conditions which may then be employed in seeing forecasts.

\section{Acknowledgements}

The authors wish to thank the following supporters of this project: Inkaba yeAfrica, National Research Foundation (NRF), Hartebeesthoek Radio Astronomy Observatory (HartRAO), University of Pretoria (UP) and Nansen Environmental and Remote Sensing Center (NERSC) as well as the referee, Dr Stoffel Fourie, for his insightful comments and suggestions. This is Inkaba yeAfrica contribution number 64 .

\section{References}

Abahamid, A., Vernin, J., Benkhaldoun, Z., Jabiri, A., Azouit, M. and Agabi, A., 2004. Seeing, outer sacle of turbulence, and coherence outer scale at different astronomical sites using instruments on meteorological balloons. Astronomy and Astrophysics, 422, 1123-1127.

André, J.C., De Moor, G., Lacarrère, P., Therry, G. and Da Vachat, R., 1978. Modeling the 24-hour evolution of the mean and turbulent structures of the planetary boundary layer. Journal of the Atmospheric Sciences, 35, 1861-1883.

Argyle, B. (Editor) 2004. Observing and Measuring Visual Double Stars. Patrick Moore's Practical Astronomy Series, Springer-Verlag, London Limited, 19 and 86pp.

Aristidi, E., Fossat, E., Agabi, A., Mékarnia, D., Jeanneaux, F., Bondoux, E., Challita, Z., Ziad, A., Vernin, J. and Trinquet, H., 2009. Dome C site testing: surface layer, free atmosphere seeing, and isoplanatic angle statistics. Astronomy and Astrophysics, 499, 955-965.

Bean, B.R. and Dutton, E.J., 1966. Radio meteorology. National Bureau of Standards Monograph, 92, 20, Superintendent of Documents, US Government Printing Office, Washington, D.C., United States of America. NBS monographs series, 92, 20pp.

Combrinck, W.L., Fourie, C.J.S., Croukamp, L. and Saunders, I., 2007. Report on preliminary geotechnical and tropospheric site investigation for a proposed space geodetic observatory near Matjiesfontein in the Great Karoo. South African Journal of Geology, 110, 225-234.

Esau, I., 2004. Simulation of Ekman boundary layers by large eddy model with dynamic mixed subfilter closure. Environmental Fluid Mechanics, 4, 273-303.

Fried, D.L., 1965. Statistics of a geometric representation of wavefront distortion. Journal of the Optical Society of America, 55, 1427-1435.

Fried, D.L., 1966. Optical resolution through a randomly inhomogeneous medium for very long and very short exposures. Journal of the Optical Society of America, 56, 1372-1379.

Garrat, J.R., 1995. The atmospheric boundary layer. Cambridge Atmospheric and Space Science Series, Cambridge University Press, Cambridge, United Kingdom, 224-226.

Kolmogorov, A.N., 1941. The local structure of turbulence in incompressible viscous fluid for very large Reynolds' numbers. Comptes rendes (Doklady) de l'Academie des Sciences de l'USSR, 30, 301-305.

Pope, S.B., 2000. Turbulent flows. Cambridge University Press, Cambridge, United Kingdom, 182pp.

Quirrenbach, A., 2006. The effects of atmospheric turbulence on astronomical observations. In: P. Cassen, T. Guillot and A Quirrenbach (Editors). A. Extrasolar planets. Saas-Fee Advanced Course 31, SpringerVerlag, Heidelberg, Germany, 137, 137pp

Roddier, F. (1981). The effects of atmospheric turbulence in optical astronomy. In: E. Wolf (Editor). Progress in optics Volume XIX. NorthHolland Publishing Co., Amsterdam, 19, 301pp.

Stull, R.B., 1988. An introduction to boundary layer meteorology. Kluwer Academic Publishers, Dordrecht, The Netherlands, 4-5 and 420-425.

Tatarski, V.I., 1961. Wave propagation in a turbulent medium. Dover Publications, New York, United States of America.

Trinquet, H. and Vernin, J., 2006. A model to forecast seeing and estimate profiles from meteorological data. Publications of the Astronomical Society of the Pacific, 118, 756-764.

Vernin, J. and Muñoz-Tuñón, C., 1992. Optical seeing at La Palma Observatory, I. General guidelines and preliminary results at the Nordic Optical Telescope. Astronomy And Astrophysics, 257, 811-816.

Editorial handling: R.B. Trumbull 